\title{
Nachrichten via Instagram, YouTube und Tiktok - Journalistische Angebote für junge Menschen
}

\author{
Martina Marx
}

\section{Zusammenfassung}

65 Prozent der jungen Menschen zwischen 18 und 24 Jahren in Österreich definieren ihre Hauptnachrichtenquelle als online. Traditionelle Medienangebote wie Print, TV und Radio erreichen diese Altersgruppe kaum. Journalistische Angebote gibt es für diese Altersgruppe auf dem österreichischen Medienmarkt wenige. In Deutschland ist dies anders, dort gibt es, finanziert von ARD und ZDF ein öffentlich-rechtliches ContentNetzwerk, das journalistische Inhalte produziert und via Sozialen Medien verbreitet. Eben dort, wo sich die junge Zielgruppe bewegt. Auch die großen Verlage unterhalten spezielle Plattformen für junge Menschen, ein Beispiel ist „bento“ vom Magazin „Der Spiegel“. Welch diverse Formate es auf dem deutschen Markt gibt und welche die größten Herausforderungen für Journalismus für junge Menschen sind, umreißt dieser Beitrag.

\section{Abstract}

65 percent of young Austrians between 18 and 24 years old define their main news source as being online. Traditional news outlets such as newspapers, TV or Radio are not capable to reach this age group any longer. Still, journalistic content, produced for this specific age group is rarely found in Austria. Meanwhile, Germany is several steps ahead. The two big public service broadcasters ARD and ZDF fund a content network called "funk", that produces content for young people and distributes this content via social media to reach young users. Almost every one of the big publishing houses also has a young spin-off, e.g. "Der Spiegel" developed "bento". This article will depict which

Katharina Gallner-Holzmann, Theo Hug, Günther Pallaver (Hg.):

Jugendliche Mediennutzung und die Zukunft des Qualitätsjournalismus

(C) 2020 innsbruck university press, ISBN 978-3-99106-014-7, DOI 10.15203/99106-014-7 
diverse content for young people there is within the German media circuit and discuss the challenges of journalism aiming at a young audience.

\section{Einleitung}

„Österreich braucht ganz dringend so etwas wie ,funk' in Deutschland. Ein paar Mio. $€$ der GIS-Gebühren p.a., um jungen Menschen öffentlich-rechtlichen Journalismus auf Instagram, YouTube, TikTok, in aufwändigeren Podcasts etc. zu präsentieren. Ein $€$-Lercherlschas mit Impact.“ Dies schrieb Journalist und Podcaster Andreas Sator Ende April 2020 auf Twitter (Sator, 2020).

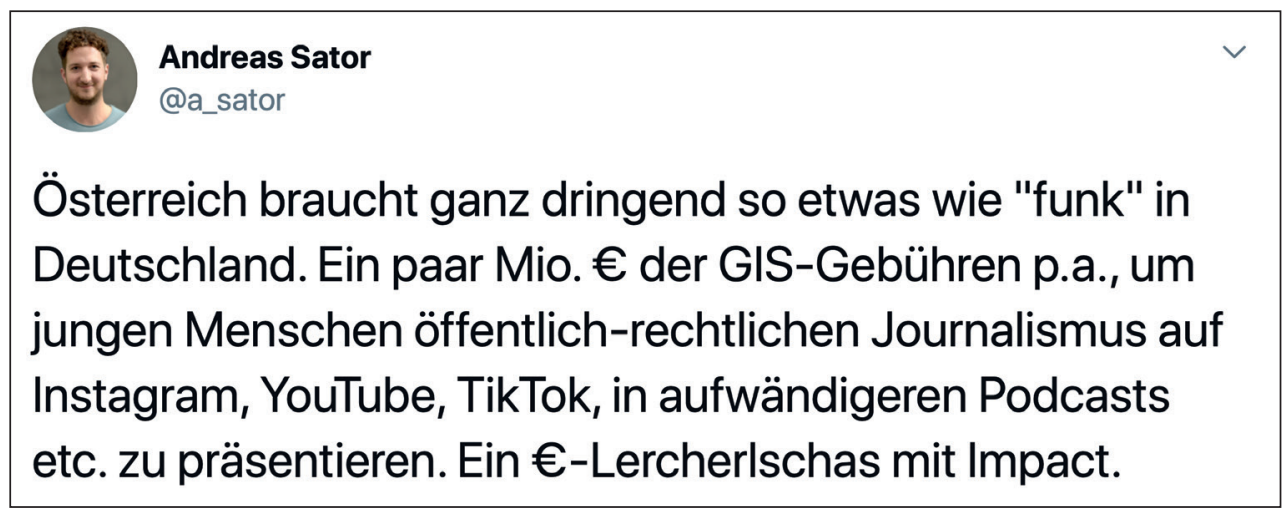

Abbildung 1: Tweet von Andreas Sator zum Medienangebot für junge Menschen in Österreich. abgesetzt am 28. April 2020 via Twitter, Screenshot.

Wieso aber braucht es Nischenprodukte, die auf eine junge Zielgruppe zugeschnitten sind, wie etwas das von Sator als Beispiel gebrachte öffentlich-rechtliche Angebot von „funk“. Die Antwort auf diese Frage ist im Mediennutzungsverhalten junger Menschen zu finden. 


\title{
Daten und Fakten zur Mediennutzung einer jungen Zielgruppe
}

Grundsätzlich definieren 65 Prozent junger Menschen zwischen 18 und 24 Jahren ihre Hauptnachrichtenquelle als online. Bei der Gruppe der 35 bis 44-Jährigen ist diese genau umgedreht, hier wird die Hauptnachrichtenquelle als traditionell definiert - also Radio, TV und Print. Betrachtet man die Altersklasse 55+, so definiert diese ihre Hauptnachrichtenquelle zu 82,2 Prozent als traditionell. Diese Erhebung stammt aus dem Reuters Digital News Report 2019 für Österreich (Gradinger et al. 2019). Hier heißt es weiters:

\begin{abstract}
„Betrachtet man die Nachrichtenquellen nach den Altersgruppen, so zeigen sich vor allem bei traditionellen Quellen wie Fernsehnachrichten, gedruckten Zeitungen und Radionachrichten signifikante Unterschiede zwischen den Generationen: Von älteren Personen werden diese Medien häufiger genutzt (z.B. TV-Nachrichten: 55+: 77,3 \% und 18-24: 36,9\%). Auf der anderen Seite sind für die 18- bis 24-Jährigen Soziale Medien die am häufigsten verwendete Nachrichtenquelle (67,3\%).“ (Gradinger et al. 2019, S. 34)
\end{abstract}

Beleuchtet man den Weg, wie User auf Nachrichten stoßen, genauer, manifestiert sich die Relevanz von Sozialen Medien für eine junge Zielgruppe noch vehementer. Über alle Altersklassen hinweg gesehen ist der hauptsächliche Weg zum Auffinden von Nachrichten der direkte über die App der Medienmarke, und zwar zu 24,7 Prozent. Auf Rang zwei folgen die Sozialen Medien mit 18,4 Prozent (Gradinger et al. 2019, S. 68). Doch analysiert man die Angaben der beiden jüngsten, für den Reuters Digital News Report definierten, Altersgruppen, ergibt sich ein gänzlich anderes Bild. Denn für diese sind die Sozialen Medien mit einigem prozentuellen Abstand die wichtigste Quelle um auf Nachrichten zu stoßen: Für 23,2 Prozent der 18- bis 24-Jährigen, sowie für 28,7 Prozent der 25- bis 34-Jährigen - siehe dazu auch Abbildung 2. 


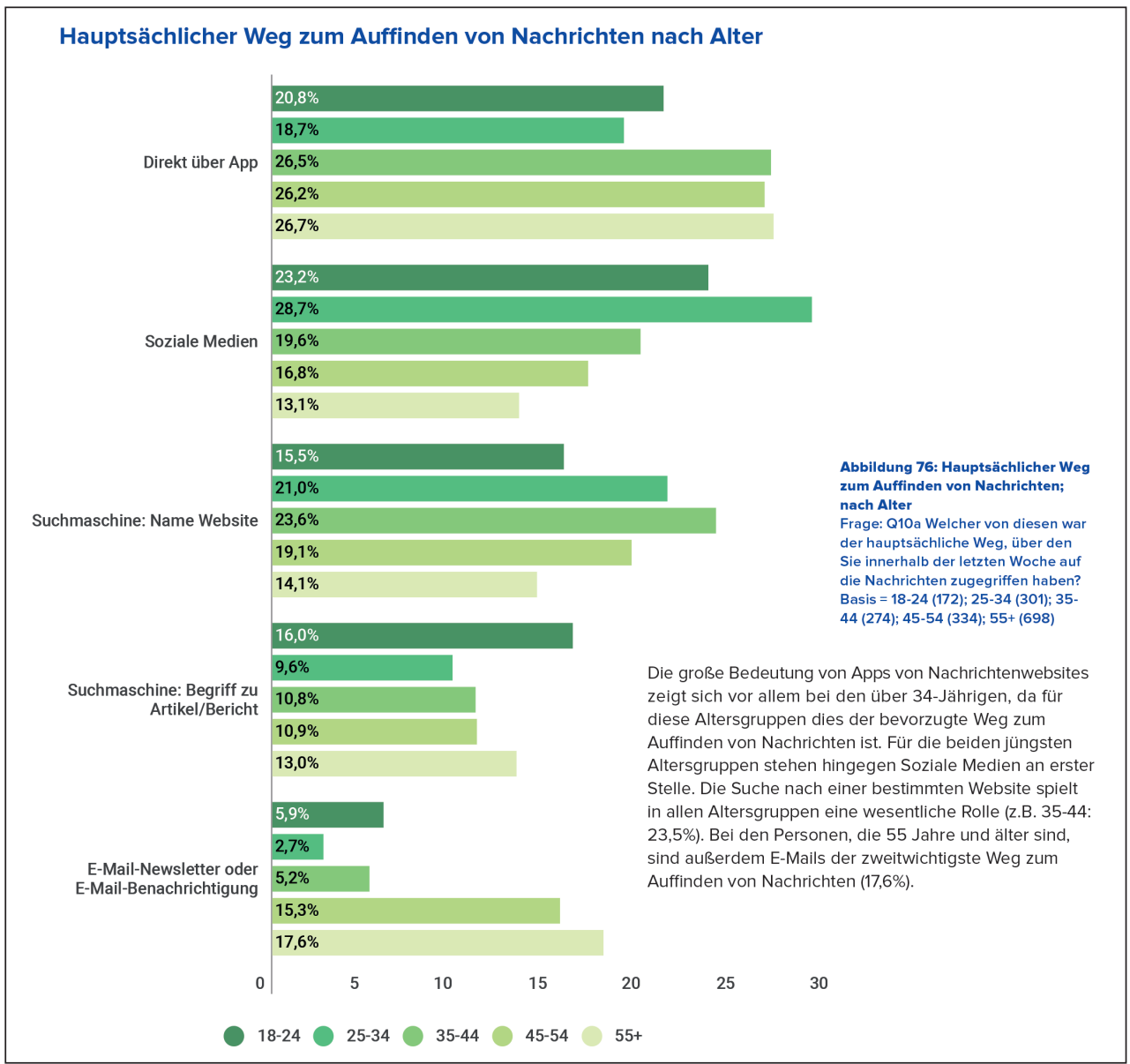

Abbildung 2: Auf welchem Weg User zu ihren Nachrichten kommen. Aus: Reuters Digital News Report 2019. Diagramm aus dem Reuters Digital News Report 2019 für Österreich, Seite 68. Screenshot.

Diese Tendenz belegt auch der „Jugend Internet Monitor 2020“ für Österreich. Im Rahmen dessen wird erhoben, welche Sozialen Medien junge Menschen zwischen elf und 17 Jahren nutzen. Im Vergleich zu 2019 sind alle Sozialen Medien in ihren Nutzungsdaten gestiegen. Am stärksten TikTok mit einer Zuwachsrate von 23 Prozent auf 42 Prozent (saferinternet.at, 2020). 


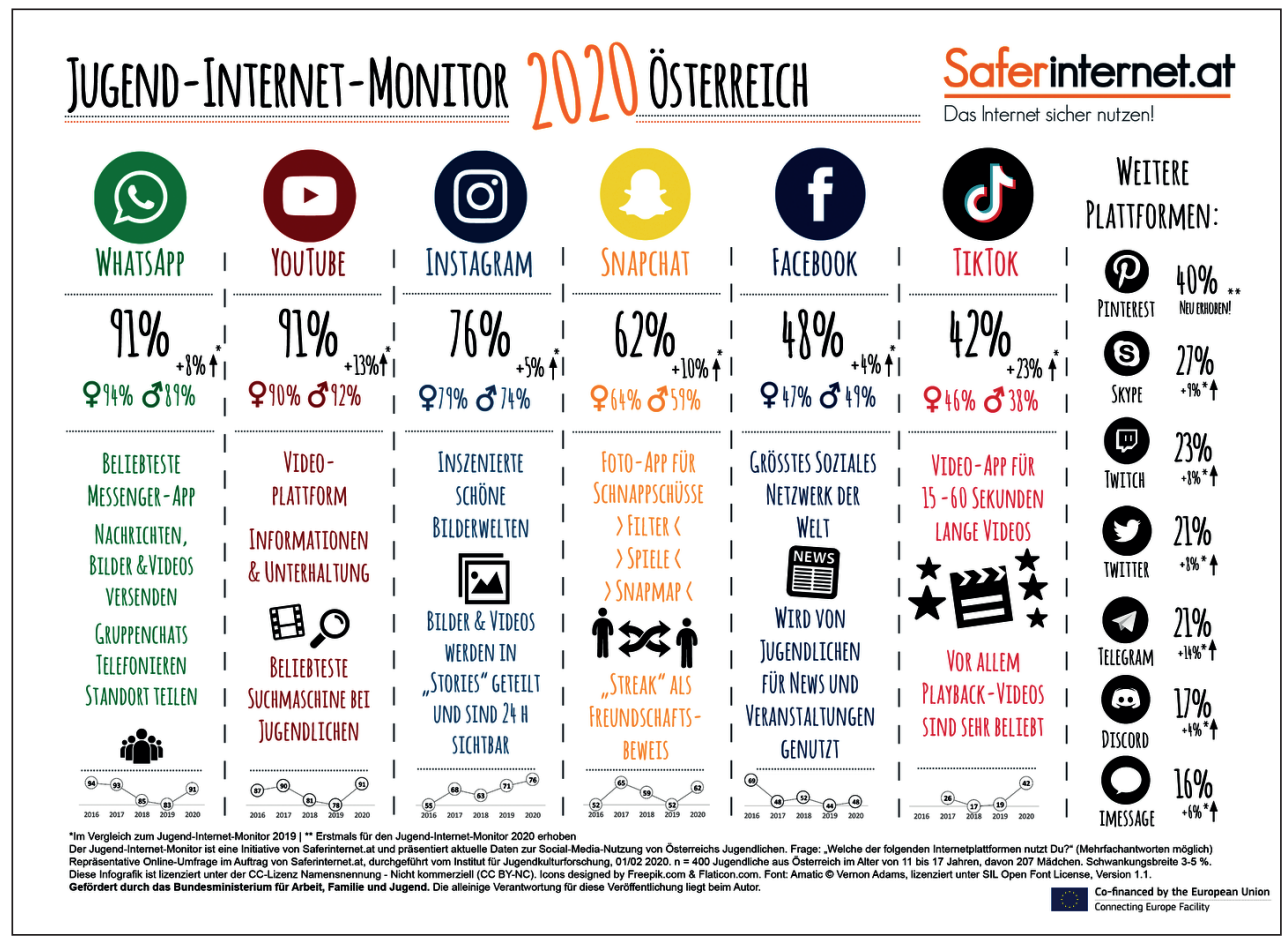

Abbildung 3: Jugend-Internet-Monitor 2020, der vom Institut für Jugendkulturforschung im Auftrag der Initiative saferinternet.at erstellt wird. Grafik zum Jugend-Internet-Monitor 2020, Screenshot.

Die Conclusio aus diesen Zahlen ist: Junge Menschen sind interessiert an Nachrichten und Medien. Sie suchen ihre Informationen online, und das vor allem in Sozialen Medien. Vor allem Facebook nutzen viele jüngere User wie eine Nachrichten-Plattform. Die Schlussfolgerung lautet, dass Medien- bzw. Verlagshäuser ihre journalistischen Inhalte dort anbieten müssen, wo sich die junge Zielgruppe bewegt. Also in den Sozialen Medien.

Denn junge Menschen kann man mit journalistischen Inhalten dort erreichen, wo sie sich selbst aufhalten. Ebendort müssen die Inhalte plattformgerecht aufbereitet sein. Die Erfahrung lehrt etwa die Autorin dieser Zeilen, dass ein Beitrag, der auf Facebook funktioniert und viel Reichweite und/oder Engagement generiert, auf Instagram gar nicht funktionieren muss. Um mit Usern authentisch und auf Augenhöhe kommunizieren zu können, muss man die Plattform, auf der man vertreten ist, verstehen und auch mit Expertise bespielen. 
Betrachtet man das aus Österreich stammende journalistische Angebot für junge Menschen, stößt man auf einige wenige Plattformen. Die Kronen Zeitung betreibt das Portal City4You, der Kurier hat im k.at im Vorjahr eine neue Medienmarke „für Millenials" erschaffen (derstandard.at, 2019). Die Autorin dieses Textes zeichnet für Futter, das junge Magazin der Kleinen Zeitung verantwortlich. Und Vice Österreich wird seit 2018 mit einer verkleinerten Redaktion von Deutschland aus geführt (Goldenberg, 2019). In der Welt der Funkwellen hält Radio FM4 die Flagge der Jugend hoch, übrigens das einzig öffentlich-rechtliche Angebot unter den hier für Österreich genannten.

\section{Das junge Medien-Angebot in Deutschland}

In Deutschland ist ein anderes, um einiges differenziertes Bild in Bezug auf journalistisches Angebot für junge Menschen zu finden. Zahlreiche der bekannteren Medienmarken unterhalten eigene Content-Angebote für eine junge Zielgruppe. So gehört etwa zum Magazin „Der Spiegel“ „,bento“, das Angebot der Wochenzeitung „Die Zeit“ nennt sich „ze.tt". Auch die „Süddeutsche Zeitung“ versucht Millenials via ,jetzt.de“ zu erreichen. Der „Stern“ betreibt „Neon“ und das „Handelsblatt“ Orange.

\section{Funk: Öffentlich-rechtlicher Content}

Hinzu kommt, wie schon eingangs erwähnt, das umfangreiche Angebot von „funk“. Die Eigendefinition der öffentlich-rechtlichen Plattform lautet wie folgt: „Wir sind funk, das Content-Netzwerk von ARD und ZDF. Unsere funk-Formate aus den Bereichen Information, Orientierung und Unterhaltung sind auf YouTube, Facebook, Snapchat, TikTok und Instagram sowie auf funk.net zu finden.“ (funk, 2020)

Funk richtet sich an die Altersgruppe der 14- bis 29-Jährigen. Da die Interessen von 14- und 29-Jährigen aber sehr divers sein können, wird versucht, mit verschiedensten Content-Formaten die unterschiedlichen Nischen der jungen Zielgruppe zu erreichen. Diese haben oft keinen eigenen Webauftritt, es können auch Accounts auf den unterschiedlichen Sozialen Medien sein. Auch Podcasts finden sich unter den ContentFormaten. Zum Portfolio gehört der feministische Instagramkanal „Mädelsabende“ - betrieben vom WDR - ebenso wie „Kliemansland“ mit Fynn Kliemann auf YouTube. Weiters umfasst das Angebot „reporter“, ein Format für Dokumentationen und Reportagen des WDR, oder „mailLab“, das Wissenschaftsjournalismus auf eine informative und unterhaltende Weise präsentiert. Der YouTube-Kanal der Chemikerin und Moderatorin Mai Thi Nguyen-Kim wurde mehrmals ausgezeichnet. Zuletzt im April 
2020 mit dem Heinz-Oberhummer-Award für Wissenschaftskommunikation der österreichischen „Science Busters“ (Pokropek, 2020).

Die Auflistung dieser Formate zeigt, dass das Content-Netzwerk die unterschiedlichsten Formate wählt. Hier haben journalistisch hochwertige Reportagen neben humoristischen Sketch-Shows ebenso Platz, wie ein Podcast über E-Gaming oder ein Instagram-Account über Naturkosmetik und Beauty. Insgesamt gibt es über 70 Kanäle und noch mehr unterschiedliche Formate (funk, 2020). Diese sind so zahlreich, weil es ,unmöglich ist, alle Personen dieser Altersgruppe mit einem einzigen Kanal zu erreichen“, wie Programmgeschäftsführer Florian Hager sagt (Pramer, 2018). Er sei davon überzeugt, „,dass die Öffentlich-Rechtlichen dorthin gehen müssen, wo die Meinungsbildung und die Diskussion um gesellschaftlich relevante Themen stattfindet“. (Pramer, 2018)

Die Inhalte des Content-Netzwerkes werden genutzt und von der Zielgruppe angenommen. Dies belegt eine gemeinsame Untersuchung von AGF Videoforschung und YouTube, der Untersuchungszeitraum war Oktober 2018. Demnach erreichten die funk-Inhalte auf YouTube 58 Millionen Views. Somit lag „funk“ vor sämtlichen YouTube-Kanälen der ARD-Familie (57 Millionen) und den ZDF-Inhalten (19 Millionen) (Schröder, 2019).

Finanziert wird „funk“ durch den Rundfunkbeitrag, das jährliche Budget beträgt laut eigenen Angaben rund 45 Millionen Euro. Zwei Drittel werden vom ARD finanziert, ein Drittel vom ZDF.

\section{News-WG: Nachrichten auf Instagram}

Auch abseits von „funk“ engagieren sich öffentlich-rechtliche Anstalten in Deutschland im Bereich junger, journalistischer Inhalte. Hervorzuheben ist hier etwa NewsWG, eine Initiative des Bayrischen Rundfunks. „News zum Verstehen \& Mitreden. Storys aus Politik, dem Drumherum \& der WG. Hintergründe statt Halbwissen." So steht es in der Beschreibung des Kanals auf Instagram (News-WG, 2020). Von den drei Volontärinnen Ann-Kathrin Wetter, Sophie von der Tann und Helene Reiner erdacht und anfangs noch belächelt, wurde die News-WG mittlerweile mit mehreren Preisen ausgezeichnet, unter anderen 2019 mit dem „Goldene Blogger“-Preis für den besten Instagram-Account (Tran, 2019).

Über 80.000 Follower verzeichnet der Account im Mai 2020. Ann-Kathrin Wetter ist von der Volontärin zur Redakteurin im News-WG-Team geworden. Die Abläufe, wie 
etwa Recherche, seien ähnlich wie in einer klassischen Redaktion, anders sei jedoch die Umsetzung: Eben nur für Instagram. Auf der Social-Media-Plattform wird vor allem mit Stories gearbeitet. 15 Sekunden lange Video- bzw. Bild-Sequenzen, die passend aneinandergereiht, spannendes und informatives Storytelling darstellen können. Oder wie Wetter es beschreibt:

„Das ist ja immer der Trugschluss, dass Instagram so oberflächlich ist. Unsere Storys sind ja nicht 15 Sekunden lang, sondern die sind teilweise sogar richtig lang, wenn man die sich anschaut, und die Leute bleiben aber trotzdem dran. Und das machen wir mit den Mitteln, allen, die Instagram bietet und mit dem Storytelling, dass wir als Journalisten im Volontariat gelernt haben." (Tran, 2019)

Beim Sehen der Stories soll das Gefühl entstehen, dass der Content spontan entstanden ist, aufgenommen in einer WG in München. Doch hinter den kurzweiligen Instagram-News stecken Planung, Recherche, Videodrehs, Schnittarbeit und viele andere Techniken, die in einem Großteil der Redaktionen von Medien stattfinden und ausgeübt werden. Durch die Präsentation auf Instagram aus der WG wirken die Inhalte authentischer, die Redakteurinnen zugänglich und auf Augenhöhe mit den Usern. Einen erhobenen Zeigefinger wird man in dieser Art der Berichterstattung vergeblich finden, dieser wäre nicht nur fehl am Platz, sondern auch kontraproduktiv.

„Was für uns ganz wichtig ist: Mut zur Imperfektion. Bei uns ist es total oft so, wenn wir an ein Thema herangehen, dass wir auch zugeben, wenn wir selber noch nie davon gehört haben. Wir wissen hier nicht alles, wir müssen uns auch erst mal daransetzen, recherchieren, und das sagen wir auch unseren Follower“, erklärt News-WG-Moderatorin Helene Reiner. (br.de, 2019)

\section{„bento”: Das junge Magazin von „Der Spiegel“}

Wie eingangs erwähnt, unterhalten zahlreiche bekannte deutsche Medienmarken eigene Produkte, um junge Menschen anzusprechen. Eines der bekanntesten ist „bento“, das junge Magazin von „Der Spiegel“, das im Oktober 2015 startete. Das Ziel war, rasch zu konsumierende News für die „Generation Hashtag“ zu produzieren, angereichert mit einer längeren Story pro Tag sowie Erklärstücken zur aktuellen Nachrichtenlage.

Das Konzept wurde angenommen, „bento“ hat sich etabliert. 2019, nach vier Jahren, wurden Website und Inhalte einem Relaunch unterzogen. „'bento“ mit seinen sehr 
guten Reichweitenwerten hängt heute zu gut drei Vierteln an spiegel.de: Wird von dort verlinkt, erreicht eine Geschichte viele interessierte Leser, ansonsten allzu oft nicht", schreibt das Development-Team des Spiegel (derspiegel, 2019) - es ist dies ein Punkt, auf den im Abschnitt „Herausforderungen“ noch näher eingegangen wird.

Das neue Konzept, mit dem „bento“ im Herbst 2019 gestartet ist, richtet sich an Menschen zwischen 18 und 30 Jahren. Es werden Geschichten erzählt, die sich an den Lebensrealitäten junger Menschen orientieren und die aus deren Sicht erzählt werden. Die Leitidee lautet: „Wir berichten über die Welt in ihrer Komplexität - aber aus junger Perspektive.“" (derspiegel, 2019)

Die redaktionelle Kraft, die hinter den jungen Magazinen in Deutschland steckt, ist mit jener in Österreich kaum vergleichbar: „bento“ beschäftigt zwischen zehn und 15 Vollzeitkräfte, ,ze.tt“ aus dem Hause „Die Zeit“ hat ein Team von rund zwanzig fixen Köpfen, das in Berlin unabhängig vom Stammhaus in Hamburg arbeitet. Bei „Futter“ arbeiten fünf Vollzeitkräfte, bei „k.at“ ist dies ähnlich.

\section{Journalismus für junge Menschen}

Grundsätzlich unterscheidet sich Journalismus für junge Menschen nicht großartig von jeder anderen Art von Journalismus. Für Jung wie Alt gilt, was einst schon Josef Pulitzer gesagt hat: „Was immer du schreibst, schreibe kurz, und sie werden es lesen. Schreibe klar, und sie werden es verstehen. Schreibe bildhaft, und sie werden es im Gedächtnis behalten."

Diese Aussage des Namensgebers des wichtigsten Journalismuspreises der Welt hat eine Studie des Center for Media Engagement der Universität Texas in Austin eindrucksvoll bestätigt. In dieser wurden User bzw. Leser_innen gefragt, was sie von Medien erwarten, welche Informationen sie benötigen, um Nachrichten und Geschichten richtig einordnen zu können.

Die Ergebnisse lauten wie folgt: Leser_innen möchten tiefer gehende Stories und Artikel lesen. Um Nachrichten und Zusammenhänge besser verstehen zu können, sollten Ausdrücke und Abläufe regelmäßig erklärt und nicht vorausgesetzt werden. Die Auswahl von Quellen soll beschrieben werden und es soll klar dargelegt werden, dass die Redaktion unabhängig von jedwedem Einfluss ist (Wilner et al. 2019) 
Für Journalist_innen, die für junge Medien arbeiten, heißt dies Erklärstücke, die mit den Usern auf Augenhöhe kommunizieren, zu verfassen bzw. zu produzieren. Es bedeutet, die Perspektiven und Lebensrealitäten der Zielgruppe in die Berichterstattung zu inkludieren. Und 2020 bedeutet dies auch, jenes Soziale Medium zu kennen und zu bespielen, auf dem sich die von dem Medium definierte Zielgruppe bewegt.

\section{„Futter“: Das junge Magazin der Kleinen Zeitung}

„Futter“, das junge Magazin der Kleinen Zeitung wurde 2015 ins Leben gerufen. Ähnlich wie bei „bento“ wurde auch bei „Futter online“ während der ersten Jahre - sehr erfolgreich - auf Clickbait-Content für schnelle Unterhaltung und Zerstreuung gesetzt. 2018 allerdings wurde auch „Futter“ einem inhaltlichen Relaunch unterzogen. Im Zuge dessen wurde eine eigenständige Content-Strategie entwickelt. Es wird nun sehr genau entschieden, welche Inhalte produziert werden und welche nicht.

Das Ziel ist es, Inhalte zu erstellen, die junge Menschen bewegen, die jungen Menschen einen Mehrwert bieten. Das Redaktionsteam versucht, die Wünsche und Interessen der User in die Berichterstattung einfließen zu lassen. Ein aktuelles Beispiel: Während der Zeit der Ausgangsbeschränkungen im Zuge der Maßnahmen gegen die Coronaviruspandemie wurden die User via Instagram-Stories befragt, was sie zu diesem Thema lesen möchten. Daraus ist etwa ein Artikel über Long-Distance-Beziehungen entstanden, die Interviewpartner_innen waren Mitglieder der „Futter“-Community (Marx, 2020).

Persönliche Ansprache in Du-Form und das Einbinden von persönlichen Erfahrungen der User aber auch der Redaktionsmitglieder, gewährleisten, dass sich „Futter“ auf Augenhöhe mit der Zielgruppe bewegt.

Dazu gehört auch, zu wissen, über welche Kanäle und Geräte User die Inhalte nutzen. Rund 80 Prozent aller Zugriffe erfolgen per Smartphone, weswegen versucht wird, das Nutzererlebnis hier zu perfektionieren. Im Frühjahr 2020 läuft aus diesem Grund ein Test mit „Newsroom Studio“, einem Tool, mit dessen Hilfe man ein ContentFormat, dass Instagram-Stories nicht unähnlich ist, erstellen und auch auf einer Website implementieren kann. So wird rasch konsumierbarer, optisch ansprechender Content zur Verfügung gestellt, der dennoch die wichtigsten Details vermittelt.

Dieser Test läuft vielversprechend, zeigt aber auch, dass man sich als Medium für eine junge, digital-affine Zielgruppe ständig weiterentwickeln muss. 
Die Coronavirus-Pandemie überschattet vieles. Aktuell auch den Kampf gegen den Klimawandel. Wir haben nachgefragt, wie Fridays for Future und Extinction Rebellion dennoch versuchen, für wirkungsvolle Klimamaßnahmen zu demonstrieren. Denn immerhin stehen der World Earth Day und der fünfte globale Klimastreik vor der Tür.

Klares Wasser und Fische in den Kanälen Venedigs, eine geringere Feinstaubbelastung in Graz oder kaum Flugbewegungen über Europa - check Flightradar24: Die Zeichen, dass die Coronavirus-Pandemie auch Auswirkungen auf die Umwelt und das Klima hat, sind vielfältig. Und natürlich erfreulich. Es gibt aber in dieser Hinsicht ein ziemlich großes Aber: Ist diese Entwicklung von Dauer? Was passiert, wenn alle Geschäfte, Unternehmen und Fabriken wieder ihre Arbeit aufnehmen? Ganz zu schweigen vom Flugbetrieb? Wird dann der Klimaschutz, der in den letzten Monaten durch Greta Thunberg, Fridays for Future und Co. einiges an Fahrt aufgenommen hat, wieder zur Seite geschoben, um die Wirtschaft zu retten?

Du willst kurz und bündig diese Story lesen? Dann click dich hier durch

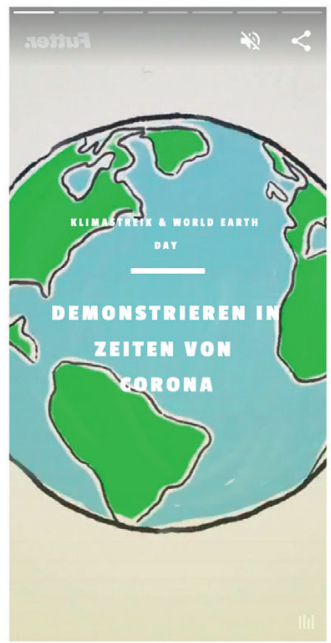

\section{Wirtschaft oder Klima?}

Die Chancen stehen - im negativen Sinn - gut. Denn schon jetzt ist klar, dass es Milliarden Euro kosten wird, die Wirtschaft wieder anzukurbeln, Unternehmen - große wie kleine - in dieser schwierigen Zeit zu unterstützen. Klar ist aber auch, dass der Kampf gegen die Klimakrise drängt. Wir haben keine Zeit, Maßnahmen zum Klimaschutz auf später zu verschieben. Denn wenn die Erderwärmung nicht mehr als 1,5 Grad Celsius bis 2050 steigen soll, dann müssen die Maßnahmen ab jetzt, ab 2020, 2021 gesetzt werden.

Abbildung 4: Newsroom Studio im Test auf der Futter-Website, Screenshot Tapable Content für eine Website, umgesetzt mit dem Tool „Newsroom Studio “ auf futter.kleinezeitung.at, Screenshot. 


\section{Herausforderungen für junge Medien}

Die Herausforderungen in Bezug auf journalistische Produkte für junge Menschen sind aus Sicht der Autorin dieses Textes vorrangig zweierlei:

\section{Technische Fertigkeiten}

Gerade wenn es um das Erstellen von Inhalten für eine sehr spitze oder enge Zielgruppe geht, sollte man als Redaktion wissen, welche Themen man im Sinne der User ansprechen soll. Was funktioniert gut, was nicht? Es ist natürlich 2020 um ein Vielfaches leichter, Erkenntnisse über seine User zu erhalten als noch vor einigen Jahren. Aber um Google Analytics und Co. aussagekräftig nutzen zu können, braucht es Wissen und Expertise um diese Analysetools.

Gleiches gilt für den Bereich der Suchmaschinenoptimierung. Inhalte müssen so aufgebaut sein, dass sie von Suchmaschinen gefunden werden können. Auch hier ist Wissen und Expertise notwendig.

Dies sind nur zwei Beispiele, die zeigen, dass sich die Profession des/r Journalist in weiterentwickelt hat. Einen guten Artikel zu schreiben reicht schlicht nicht mehr. Als Journalist_in muss man wissen, wie man seine Zielgruppe richtig bedient, man muss Storytelling beherrschen, die Umsetzung in den Sozialen Medien mitdenken und auch die technischen Fertigkeiten wie SEO oder den Umgang mit Analyse-Tools beherrschen. Hier besteht für die Verlagshäuser Schulungsbedarf ihrer Mitarbeiter_innen. Denn nur so können Angebote für eine junge Zielgruppe entwickelt werden und Medienmarken als solche relevant bleiben.

\section{Wirtschaftlichkeit}

Verlagshäuser haben erkannt, dass sie ein Angebot für junge Leser_innen anbieten müssen bzw. sollten. Der Hintergrund dieser Erkenntnis: Wirtschaftlichkeit. Durch das Heranführen an die etablierte Medienmarke, etwa Kleine Zeitung, einer jüngeren Zielgruppe mit dem Vehikel eines Nischenangebots, etwa „Futter“, wird versucht die Abonnent_innen von morgen zu gewinnen. Eine Bindung an die Medienmarke soll erfolgen (Kappacher, Atzara, 2018). Vielfach wird auch versucht, durch Native Advertising bzw. Werbeformate in den Sozialen Medien neue Erlöswege zu erschließen. Doch selten sind neue Medien von Beginn an wirtschaftlich. Vor allem der Be- 
reich der Sozialen Medien ist schnelllebig, ebenso rasch müssen die Content-Formate angepasst werden.

Dies bringt mit sich, dass es für junge, journalistische Medienmarken schwierig ist, sich finanziell selbst zu tragen. Investitionen in diese rechnen sich wohl erst nach Jahren. Umso wichtiger sind Verlagshäuser, die die Weitsicht haben, qualitativ hochwertige Medienmarken abseits der traditionellen Verbreitungskanäle zu entwickeln, diese zu finanzieren um ihren künftigen Leser_innen, User_innen bzw. Seher_innen auf Augenhöhe begegnen zu können.

Womit sich der Kreis an dieser Stelle schließt: ARD und ZDF zeigen vor, wie journalistische Produktentwicklung funktionieren kann. Andreas Sator hat mit eingangs erwähnten Tweet gnz recht: Ein ähnliches Angebot braucht es auch in Österreich. Es wäre ein richtiger Schritt, um junge Menschen mit qualitativ hochwertigen journalistischen Inhalten wirklich erreichen zu können sowie durch Schulungen und Minderung des wirtschaftlichen Drucks ein solches Angebot überhaupt divers aufbauen zu können.

\section{Literatur}

Atzara, Rosanna; Stefan Kappacher (2018): Wie Verlage um junges Publikum kämpfen. Abgerufen unter: https://oe1.orf.at/artikel/640059/Wie-Verlage-um-junges-Publikum-kaempfen [Stand vom 03-05-2020].

Br.de (2019): Instagram, die Plattform für News. Abgerufen unter: https://www.br.de/ nachrichten/netzwelt/instagram-die-plattform-fuer-news,RTAj5Db [Stand vom 0403-2020].

Dev Spiegel (2019): Wie sich bento neu erfinden wird. Abgerufen unter: https://medium.com/@,devspiegel/wie-sich-bento-neu-erfinden-wird-9eb13f4ab4f [Stand vom 04-03-2020].

Der Standard (2019): Telekurier präsentiert Portal "k.at" für junge Zielgruppe. Abgerufen unter: https://www.derstandard.at/story/2000105392883/telekurier-praesentiertportal-kat-fuer-junge-zielgruppe [Stand vom 27-04-2020].

Funk (2020): Hey, wir sind funk. Abgerufen unter: https://www.funk.net/funk [Stand vom 01-05-2020].

Gadringer, Stefan; Holzinger, Roland; Nening, Isabella; Sparviero, Sergio und Trappel, Josef (2019): Reuters Digital News Report Detailergebnisse für Österreich. Abgerufen unter: http://www.digitalnewsreport.at/reports/2019-2/ [Stand vom 27-04-2020].

Goldenberg, Anna (2019): Abgerufen unter: https://www.falter.at/zeitung/20190227/ schon-wieder-neue-plaene-fuer-vice-austria/479b04188b [Stand vom 01-05-2020]. 
Saferinternet.at (2020): Jugend-Internet-Monitor 2020. Abgerufen unter: https://www. saferinternet.at/presse-detail/jugend-internet-monitor-2020-das-sind-die-beliebtesten-sozialen-netzwerke/ [Stand vom 27-04-2020].

Marx, Martina (2020): Long-Distance-Liebe in Zeiten von Corona: „Wenn du nicht vertraust, funktioniert es nicht“. Abgerufen unter: https://futter.kleinezeitung.at/ long-distance-liebe-corona/ [Stand vom 03-05-2020].

Pokropek, Benjamin (2020): Abgerufen unter: https://sciencebusters.at/heinz-oberhummer-award-2020/, [Stand vom 01-05-2020].

Pramer, Philip (2018): Abgerufen unter: https://www.derstandard.at/story/200008 $\underline{8451557 / \text { funk-geschaeftsfuehrer-oeffentlich-rechtliche-muessen-dorthin-gehen- }}$ wo-die-diskussion [Stand vom 25-04-2020].

Schröder, Jens (2019): AGF veröffentlicht erstmals Youtube-Daten: 33 Minuten Nutzung pro Tag, funk stärker als die gesamte ARD. Abgerufen unter: https://meedia. de/2019/03/06/agf-veroeffentlicht-erstmals-youtube-daten-33-minuten-nutzungpro-tag-funk-staerker-als-die-gesamte-ard/ [Stand vom 25-04-2020].

Tran, Ahn (2019): Nachrichten aus der WG-Küche - Journalismus auf Instagram. Abgerufen unter: https://www.deutschlandfunk.de/journalismus-auf-instagram-nachrichten-aus-der-wg-kueche.2907.de.html?dram:article id=451819 [Stand vom 2704-2020].

Wilner, Tamar, Montiel Valle, Dominique A., und Chen, Gina Masullo (2019, October): What people want to know about the news. Abgerufen unter: https://mediaengagement.org/research/what-people-want-to-know [Stand vom 04-03-2020]. 